\title{
Constraining star cluster disruption mechanisms
}

\author{
I. S. Konstantopoulos, ${ }^{1,2, *}$ N. Bastian, ${ }^{3}$ M. Gieles ${ }^{2}$ and \\ H. J. G. L. M. Lamers ${ }^{4}$ \\ ${ }^{1}$ Department of Physics \& Astronomy, University College London, Gower Street, London, \\ WC1E 6BT, UK \\ ${ }^{2}$ European Southern Observatory, Casilla 19001, Santiago 19, Chile \\ ${ }^{3}$ Institute of Astronomy, University of Cambridge, Madingley Road, Cambridge, CB3 0HA, UK \\ ${ }^{4}$ Astronomical Institute, Utrecht University, Princetonplein 5, 3584 CC Utrecht, \\ the Netherlands \\ ${ }^{*}$ Currently at Penn State University; email: iraklis@astro.psu.edu
}

\begin{abstract}
Star clusters are found in all sorts of environments, and their formation and evolution is inextricably linked to the star-formation process. Their eventual destruction can result from a number of factors at different times, but the process can be investigated as a whole through the study of cluster age distributions. Observations of populous cluster samples reveal a distribution following a power law of index approximately -1 . In this work, we use M33 as a test case to examine the age distribution of an archetypal cluster population and show that it is, in fact, the evolving shape of the mass detection limit that defines this trend. That is to say, any magnitude-limited sample will appear to follow a $\mathrm{d} N / \mathrm{d} \tau=\tau^{-1}$ relation, while cutting the sample according to mass gives rise to a composite structure, perhaps implying a dependence of the cluster disruption process on mass. In the context of this framework, we examine different models of cluster disruption from both theoretical and observational perspectives.
\end{abstract}

Keywords. galaxies: star clusters, galaxies: individual (M 33)

\section{Introduction}

Star clusters are commonly used to trace the stellar content and star-formation history of their host systems. The main limitations to this approach are the finite lifetime of a cluster (disruption) and evolutionary fading. From a theoretical point of view, the lifetime of a cluster should depend on its initial mass and the properties of the environment in which it evolves (Spitzer 1958†; Baumgardt \& Makino 2003). Observations have, however, produced two empirical disruption laws:

- Mass-dependent disruption (MDD; Boutloukos \& Lamers 2003: BL03);

- Mass-independent disruption (MID; Fall et al. 2005).

In the MID model, 'survivors' are selected on a purely random basis and a constant fraction is destroyed every age dex. Intriguing as it may be, this model clashes with several principles of cluster dynamics that would need to be revised considerably to accommodate it. In this contribution, we test cluster dissolution and attempt to disentangle it from incompleteness and the statistical biases that have (in the past) limited such studies. We then compare theory to observations of the M33 cluster system.

$\dagger$ This work showed that the lifetime due to encounters with interstellar clouds depends on the cluster density. Observations of young clusters have shown the radius to scatter tightly about $3.5 \mathrm{pc}$ and can thus be considered a constant for any practical formulation. This immediately establishes the cluster mass as the main deciding factor for the lifetime of a cluster. 

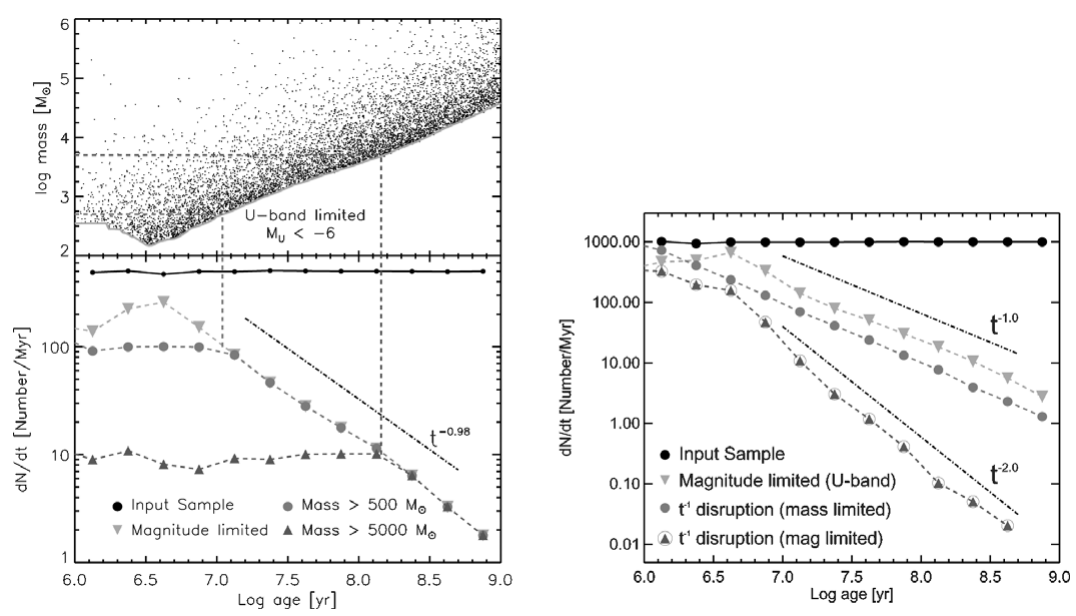

Figure 1. (left) Analysis of an artificial cluster population at the distance of M33. The population contains $5 \times 10^{5}$ clusters, sampled from a power-law mass function with index -2 , and assuming a constant cluster-formation rate. The top panel shows the mass distribution for the observable part of the population. The detection limit is denoted by the solid line. This takes into account evolutionary fading and the sample is assumed to be limited by the $U$ band, as is most commonly the case in extragalactic studies. The bottom panel shows the number of clusters per logarithmic age bin $(\mathrm{d} N / \mathrm{d} \tau)$. The dark solid line corresponds to all clusters 'formed' (i.e., the entire simulated set), while the dashed green line tracks 'observed' clusters, i.e., those above the detection limit. The theoretical expectation is for a magnitude-limited sample to follow a simple power-law distribution, while the introduction of a mass limit segments the distribution into a flat and a power-law part (the transition is dictated by the mass cut and the point where incompleteness sets in). (right) A similar population, according to the predictions of MID. This presents the age distribution of a cluster population with a constant cluster-formation rate and an imposed a $\tau^{-1}$ disruption law.

\section{A theoretical preamble}

Before providing a treatment of an observed data set, let us try to understand the 'tools of the trade', the statistical distributions used to study and characterise the cluster disruption process. Therefore, we have created an artificial population with a constant cluster-formation history $(\mathrm{CFH})$ and masses drawn from a power-law distribution with index -2 , although we note that the cluster initial mass function was recently found to be better described by a Schechter function (Gieles 2009; Larsen 2009). This population is presented in the left-hand panel of Figure 1.

\subsection{Age-mass diagram}

This plot simply shows the number of clusters with increasing age. Logarithmic bins accentuate the increase of the number of high-mass clusters with increasing age. This arises because the sample grows with time: a 'size-of-sample' effect. As the sample increases, so does the likelihood of producing a massive cluster.

\subsection{Detection limit}

Unlike the top envelope of the age-mass diagram, the line along the bottom of the distribution is not caused by statistics, but it is the detection limit of our modelled observations. As clusters fade with time, this constant limiting flux translates into an increasing limiting mass.

In most cases, observed cluster samples will be luminosity limited. As the population ages, clusters of higher mass fade to below the detection limit. We have denoted this 
by a green line, representing the mass of a cluster with $M_{U}=-6$ mag (adapted to the study of M33 that will follow) at a given age. The $U$ band is normally the limiting filter in observational studies and we emulate the detection limit in this plot.

\subsection{Age distribution: the $\mathrm{d} N / \mathrm{d} \tau$ plot}

The lower panel shows the main diagnostic used in the study of cluster disruption. The vertical axis gives the number of clusters our imaginary galaxy formed in each log(age) bin, normalised to unit time (in this case, $1 \mathrm{Myr}$ ). Here, we present the input sample as a solid line; a constant CFH gives rise to a flat line. This will not, however, be observed, due to the detection limit.

\subsection{A magnitude-limited sample}

The green line shows all clusters that lie above the detection limit in each log(age) bin and displays the characteristic power-law shape found in all observational studies. The interpretation of this plot is far from trivial. This $\tau^{-1}$ shape means that $90 \%$ of the population is lost with each age dex. MID interprets this as $90 \%$ of clusters dissolving each age dex (e.g., in M33; Sarajedini \& Mancone 2007). In this example, however, this is due to detection incompleteness. Thus, a model has to treat fading and dissolution as two separate and concurrent causes of cluster disappearance.

\subsection{Taking mass cuts}

Having established the above, we can now perform a simple test for the mass dependence of cluster disruption: cutting the sample according to mass. In the $\mathrm{d} N / \mathrm{d} \tau$ plot we present two mass cuts as a red and blue line, respectively. Both cuts split the power-law distribution into a composite shape: a flat part, i.e., a constant cluster-formation rate, and a power-law decline, due to clusters fading to below the detection limit.

\section{Observational study}

After understanding the caveats inherent in the study of the $\mathrm{d} N / \mathrm{d} \tau$ plot, we can proceed to plot the distribution of an observed cluster sample. We obtained the age and mass of a sample of $\sim 350$ bona fide clusters in M33 (covering the entire surface of the galaxy, an unbiased, HST-selected sample; Sarajedini \& Mancone 2007; San Roman et al. 2009) using $U B V I$ imaging from the Local Group Survey (Massey et al. 2006). The star-formation rate is known to have been constant for $\sim 1$ Gyr in M33, making it ideal for our study. Figure 2 shows the age distribution of the magnitude-limited sample (left) and a mass cut (right). It exhibits the same shape as the $\mathrm{d} N / \mathrm{d} \tau$ plot of Figure 1 (left), with a flat part leading to a power law.

We also provide a direct test of MID in Figure 1 (right panel), where we create a population that destroys $90 \%$ of its clusters each age dex and is subject to evolutionary fading.

\subsection{Observed $\mathrm{d} N / \mathrm{d} \tau$ plot}

To dampen the effect of local overdensities in the $\mathrm{d} N / \mathrm{d} \tau$ plot (caused by uncertainties in the simple stellar population models), in Figure 2 we represent each age by a Gaussian, where the wings are defined by the uncertainty. The result is consistent with a $\tau^{-1}$ power law for a magnitude-limited sample. Crucially, as predicted by the models of Figure 1 (left), taking a mass cut produces a composite structure, with a flat initial part and a power-law decline at older ages. This demonstrates that an observed sample needs to be interpreted as the result of fading and dissolution on different timescales. 

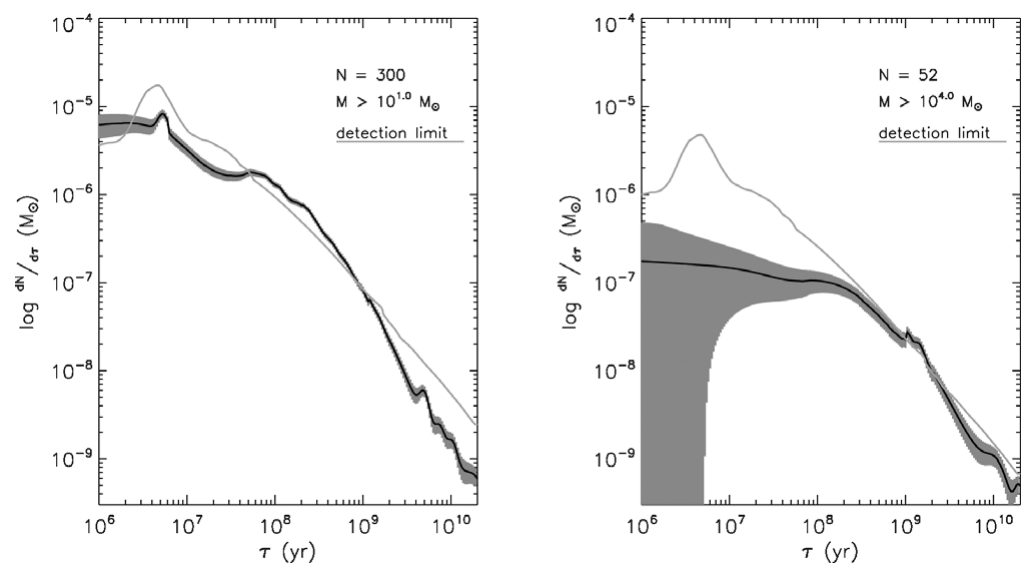

Figure 2. (left) A 'smoothed' d $N / \mathrm{d} \tau$ plot. We represent each measurement as a Gaussian, where the peak occurs at the best-fit value (from $3 \mathrm{DEF}$ ) and the wings are shaped after the uncertainty in the age fit (defined as the extrema calculated by 3DEF). This minimises the effect of uncertain measurements on the shape of the distribution, as an erratic measurement will be represented by a flat Gaussian. The solid green line is a prediction of the $\mathrm{d} N / \mathrm{d} \tau$ of a luminosity-limited sample. It is the detection limit of Figure 1 (left), inverted to reflect the maximum expected number of detections, given the detection limit (due to the complex scaling of the $\mathrm{d} N / \mathrm{d} \tau$ plot we have normalised the two lines at $1 \mathrm{Gyr}$ ). (right) The same plot with an imposed mass cut at $10^{4} \mathrm{M}_{\odot}$. The obvious flattening with respect to the magnitude-limited sample on the left strongly supports a mass dependence in the cluster disruption process (consistent with BL03).

\subsection{Mass-independent dissolution plus fading}

We have argued so far that the observed $\mathrm{d} N / \mathrm{d} \tau$ plot will show a combination of disruption and fading. The right-hand panel of Figure 1 shows the combined effect of $90 \%$ dissolution (the MID hypothesis) and $90 \%$ disappearance due to fading. This results in a very steep power-law decline (blue line) that is inconsistent with the observations of Figure 2.

This result stands firmly against the MID cluster disruption model and implies the existence of a mass dependence.

\section{Summary}

Sample incompleteness causes a $\tau^{-1}$ decline in the observed age distribution of cluster populations. Size-of-sample effects and thorough statistical methods can help to interpret the disruption process at play. The fundamental difference between magnitude- and masslimited samples can then be used to discover the underlying physics. Our results based on the nearby population of M33 clusters are inconsistent with mass-independent disruption of a fixed fraction of clusters per age dex.

\section{Acknowledgements}

ISK gratefully acknowledges the support of an ESO studentship for the undertaking of this work and would like to thank the Vitacura staff and students for their hospitality. Thanks are also due to the organising committee of the General Assembly and Symposium 266. Obrigado Rio!

\section{References}

Baumgardt, H. \& Makino, J. 2003, MNRAS, 340, 227

Boutloukos, S. G. \& Lamers, H. J. G. L. M. 2003, MNRAS, 338, 717 
Fall, S. M., Chandar, R., \& Whitmore, B. C. 2005, ApJ (Letters), 631, L133

Larsen, S. S. 2009, A\& A, 503, 467

Gieles, M. 2009, MNRAS, 394, 2113

Sarajedini, A. \& Mancone, C. L. 2007, AJ, 134, 447

San Roman, I., Sarajedini, A., Garnett, D. R., \& Holtzman, J. A. 2009, ApJ, 699, 839

Massey, P., Olsen, K. A. G., Hodge, P. W., Strong, S. B., Jacoby, G. H., Schlingman, W., \& Smith, R. C. 2006, $A J, 131,2478$

Spitzer Jr., L. J. 1958, ApJ, 127, 17 